TRANSACTIONS OF THE

AMERICAN MATHEMATICAL SOCIETY

Volume 358, Number 6, Pages 2625-2635

S 0002-9947(05)04023-7

Article electronically published on October 31, 2005

\title{
INEQUALITIES FOR EIGENVALUES OF A CLAMPED PLATE PROBLEM
}

\author{
QING-MING CHENG AND HONGCANG YANG
}

Abstract. Let $D$ be a connected bounded domain in an $n$-dimensional Euclidean space $\mathbb{R}^{n}$. Assume that

$$
0<\lambda_{1}<\lambda_{2} \leq \cdots \leq \lambda_{k} \leq \cdots
$$

are eigenvalues of a clamped plate problem or an eigenvalue problem for the Dirichlet biharmonic operator:

$$
\left\{\begin{array}{l}
\Delta^{2} u=\lambda u, \text { in } D \\
\left.u\right|_{\partial D}=\left.\frac{\partial u}{\partial n}\right|_{\partial D}=0
\end{array}\right.
$$

Then, we give an upper bound of the $(k+1)$-th eigenvalue $\lambda_{k+1}$ in terms of the first $k$ eigenvalues, which is independent of the domain $D$, that is, we prove the following:

$$
\lambda_{k+1} \leq \frac{1}{k} \sum_{i=1}^{k} \lambda_{i}+\left[\frac{8(n+2)}{n^{2}}\right]^{1 / 2} \frac{1}{k} \sum_{i=1}^{k}\left[\lambda_{i}\left(\lambda_{k+1}-\lambda_{i}\right)\right]^{1 / 2} .
$$

Further, a more explicit inequality of eigenvalues is also obtained.

\section{INTRODUCTION}

Let $\mathbb{R}^{n}$ denote an $n$-dimensional Euclidean space and let $D$ be a connected bounded domain in $\mathbb{R}^{n}$. An eigenvalue problem of a fixed membrane or Dirichlet Laplacian on a bounded domain $D$ in $\mathbb{R}^{n}$ is the following:

$$
\left\{\begin{array}{l}
\Delta u=-\lambda u, \text { in } D \\
\left.u\right|_{\partial D}=0,
\end{array}\right.
$$

where $\Delta$ is the Lapalacian in $\mathbb{R}^{n}$.

It is well known that this problem has a real and purely discrete spectrum

$$
0<\lambda_{1}<\lambda_{2} \leq \lambda_{3} \leq \cdots \rightarrow \infty
$$

Received by the editors December 10, 2002 and, in revised form, July 13, 2004.

2000 Mathematics Subject Classification. Primary 35P15, 58G25.

Key words and phrases. Eigenvalue, inequality of eigenvalue, biharmonic operator, clamped plate problem.

The first author's research was partially supported by a Grant-in-Aid for Scientific Research from the Japan Society for the Promotion of Science.

The second author's research was partially supported by the NSF of China and the Fund of CAS.

(C)2005 American Mathematical Society Reverts to public domain 28 years from publication 
Here each eigenvalue is repeated from its multiplicity. When $n=2$, in 1955 and 1956, Payne, Pólya and Weinberger proved that, in [10] and [11,

$$
\frac{\lambda_{2}}{\lambda_{1}} \leq 3 \text { for } D \subset \mathbb{R}^{2}
$$

and they conjectured

$$
\frac{\lambda_{2}}{\lambda_{1}} \leq\left.\frac{\lambda_{2}}{\lambda_{1}}\right|_{\text {disk }} \approx 2.5387
$$

with equality if and only if $D$ is a disk. For general $n \geq 2$, an analogous statement is

$$
\frac{\lambda_{2}}{\lambda_{1}} \leq 1+\frac{4}{n} \quad \text { for } D \subset \mathbb{R}^{n},
$$

and the conjecture of Payne, Pólya and Weinberger is

$$
\frac{\lambda_{2}}{\lambda_{1}} \leq\left.\frac{\lambda_{2}}{\lambda_{1}}\right|_{n \text {-ball }}
$$

with equality if and only if $D$ is an $n$-ball. In their excellent papers [3, 44 and [5], Ashbaugh and Benguria solved this important conjecture of Payne, Pólya and Weinberger.

On the other hand, for estimates of higher eigenvalues, Payne, Pólya and Weinberger in [11] proved

$$
\lambda_{m+1}-\lambda_{m} \leq \frac{2}{m} \sum_{i=1}^{m} \lambda_{i}, m=1,2, \cdots,
$$

for $D \subset \mathbb{R}^{2}$. For general $n \geq 2$, we have

$$
\lambda_{m+1}-\lambda_{m} \leq \frac{4}{m n} \sum_{i=1}^{m} \lambda_{i}, m=1,2, \cdots,
$$

for $D \subset \mathbb{R}^{n}$. Although these results introduced by Payne, Pólya and Weinberger have been extended by many authors, a result of Hile and Protter in [7] and a result of the second author in 12 are two main developments. Namely, in 1980, Hile and Protter [7] proved

$$
\sum_{i=1}^{m} \frac{\lambda_{i}}{\lambda_{m+1}-\lambda_{i}} \geq \frac{m n}{4}, \text { for } m=1,2, \cdots .
$$

It is not hard to check that the inequality (1.4) of Hile and Protter is sharper than the inequality (1.3) of Payne, Pólya and Weinberger. In 1991, Yang [12] obtained very sharp inequalities, that is, he derived

$$
\sum_{i=1}^{m}\left(\lambda_{m+1}-\lambda_{i}\right)\left(\lambda_{m+1}-\left(1+\frac{4}{n}\right) \lambda_{i}\right) \leq 0, \text { for } m=1,2, \cdots .
$$

According to the inequality, we can infer

$$
\lambda_{m+1} \leq \frac{1}{m}\left(1+\frac{4}{n}\right) \sum_{i=1}^{m} \lambda_{i}, \text { for } m=1,2, \cdots .
$$

It is easy to prove that Yang's inequalities (1.5) and (1.6) are sharper than the inequality (1.4) of Hile and Protter (see [1] and [2] for details). 
On the other hand, in order to describe vibrations of a clamped plate, we must consider an eigenvalue problem for Dirichlet biharmonic operator, called a clamped plate problem:

$$
\left\{\begin{array}{l}
\Delta^{2} u=\lambda u, \quad \text { in } D \\
\left.u\right|_{\partial D}=\left.\frac{\partial u}{\partial n}\right|_{\partial D}=0
\end{array}\right.
$$

where $\Delta$ is the Laplacian in $\mathbb{R}^{n}$ and $\Delta^{2}$ is the biharmonic operator in $\mathbb{R}^{n}$.

For this clamped plate problem, in 1956, Payne, Pólya and Weinberger [1] also established an inequality for the biharmonic operator $\Delta^{2}$. They obtained

$$
\lambda_{k+1}-\lambda_{k} \leq \frac{8(n+2)}{n^{2}} \frac{1}{k} \sum_{i=1}^{k} \lambda_{i}
$$

As a generalization of their result, in 1984, Hile and Yeh [8] obtained

$$
\sum_{i=1}^{k} \frac{\lambda_{i}^{1 / 2}}{\lambda_{k+1}-\lambda_{i}} \geq \frac{n^{2} k^{3 / 2}}{8(n+2)}\left(\sum_{i=1}^{k} \lambda_{i}\right)^{-1 / 2}
$$

by making use of an improved method of Hile and Protter 7]. Furthermore, in 1990, Hook 9, Chen and Qian 6] proved, independently, the following inequality:

$$
\frac{n^{2} k^{2}}{8(n+2)} \leq\left[\sum_{i=1} \frac{\lambda_{i}^{1 / 2}}{\lambda_{k+1}-\lambda_{i}}\right] \sum_{i=1}^{k} \lambda_{i}^{1 / 2}
$$

Recently, in [1], a survey paper on recent developments of eigenvalue problems, Ashbaugh pointed out whether one can establish inequalities for eigenvalues of the vibrating clamped plate problem which are analogous inequalities of Yang in the case of the eigenvalue problem of the Laplacian with Dirichlet boundary condition.

In this paper, we shall give an affirmative answer for the problem introduced by Ashbaugh, that is, we obtain the following:

Theorem 1. Let $\lambda_{i}$ denote the $i$-th eigenvalue of the clamped plate problem

$$
\left\{\begin{array}{l}
\Delta^{2} u=\lambda u, \quad \text { in } D \\
\left.u\right|_{\partial D}=\left.\frac{\partial u}{\partial n}\right|_{\partial D}=0
\end{array}\right.
$$

where $D$ is a connected bounded domain in $\mathbb{R}^{n}$. Then we have

$$
\lambda_{k+1}-\frac{1}{k} \sum_{i=1}^{k} \lambda_{i} \leq\left[\frac{8(n+2)}{n^{2}}\right]^{1 / 2} \frac{1}{k} \sum_{i=1}^{k}\left[\lambda_{i}\left(\lambda_{k+1}-\lambda_{i}\right)\right]^{1 / 2} .
$$

From Theorem 1, we can conclude the following more explicit inequality which is weaker than (1.12). 
Corollary 1. Under the assumption of Theorem 1, we have

$$
\begin{aligned}
& \lambda_{k+1} \leq\left[1+\frac{4(n+2)}{n^{2}}\right] \frac{1}{k} \sum_{i=1}^{k} \lambda_{i} \\
& +\left\{\left[\frac{4(n+2)}{n^{2}} \frac{1}{k} \sum_{i=1}^{k} \lambda_{i}\right]^{2}-\frac{8(n+2)}{n^{2}} \frac{1}{k} \sum_{i=1}^{k}\left(\lambda_{i}-\frac{1}{k} \sum_{j=1}^{k} \lambda_{j}\right)^{2}\right\}^{1 / 2} .
\end{aligned}
$$

Remark 1. It is obvious that inequalities (1.12) and (1.13) are sharper than the inequality

$$
\lambda_{k+1} \leq\left[1+\frac{8(n+2)}{n^{2}}\right] \frac{1}{k} \sum_{i=1}^{k} \lambda_{i} .
$$

It is easy to see that inequality (1.14) is better than inequality (1.8) of Payne, Pólya and Weinberger. We shall also discuss the relation between inequality (1.14) and inequality (1.10) introduced by Hook [9, and Chen and Qian [6] in the Remark 2 of Section 2.

\section{PRoOfs of MAIN RESUlTS}

In this section, we shall prove our main results.

Proof of Theorem 1. Let $g=x^{p}, p=1, \cdots, n$, where $\left(x^{1}, x^{2}, \cdots, x^{n}\right)$ are the standard Euclidean coordinates. Let $u_{i}$ be $i$-th orthonormal eigenfunction corresponding to eigenvalue $\lambda_{i}, i=1,2, \cdots, k$, that is, $u_{i}$ satisfies

$$
\left\{\begin{array}{l}
\Delta^{2} u_{i}=\lambda_{i} u_{i}, \quad \text { in } D \\
\left.u_{i}\right|_{\partial D}=\left.\frac{\partial u_{i}}{\partial n}\right|_{\partial D}=0, \\
\int_{D} u_{i} u_{j}=\delta_{i j}, \text { for any } i, j .
\end{array}\right.
$$

Defining a function $\varphi_{i}$ by

$$
\varphi_{i}=g u_{i}-\sum_{j=1}^{k} a_{i j} u_{j},
$$

where $a_{i j}=\int_{D} g u_{i} u_{j}=a_{j i}$, then we have

$$
\int_{D} u_{j} \varphi_{i}=0, \quad \text { for any } i, j=1, \cdots, k .
$$

Hence, we have

$$
\lambda_{k+1} \leq \frac{\int_{D}\left(\Delta \varphi_{i}\right)^{2}}{\int_{D}\left(\varphi_{i}\right)^{2}}
$$

From the definition of $g$, we have

$$
\nabla g=(0, \cdots, 0,1,0, \cdots, 0),
$$

where $\nabla$ denotes the gradient operator of $\mathbb{R}^{n}$. 
Next, we shall make an estimate of $\int_{D}\left(\Delta \varphi_{i}\right)^{2}$. From (2.1), (2.2) and (2.3), we obtain

$$
\begin{aligned}
& \int_{D}\left(\Delta \varphi_{i}\right)^{2}=\int_{D} \varphi_{i} \Delta^{2} \varphi_{i} \\
= & \int_{D} \varphi_{i}\left\{\Delta^{2}\left(g u_{i}-\sum_{j=1}^{k} a_{i j} u_{j}\right)\right\} \\
= & \int_{D} \varphi_{i}\left(\Delta^{2}\left(g u_{i}\right)-\sum_{j=1}^{k} a_{i j} \lambda_{j} u_{j}\right) \\
= & \int_{D} \varphi_{i}\left(4\left\langle\nabla g, \nabla\left(\Delta u_{i}\right)\right\rangle+\lambda_{i} g u_{i}\right) \\
= & \int_{D}\left\{4\left(g u_{i}-\sum_{j=1}^{k} a_{i j} u_{j}\right)\left\langle\nabla g, \nabla\left(\Delta u_{i}\right)\right\rangle+\varphi_{i} \lambda_{i} g u_{i}\right\} \\
= & \lambda_{i}\left\|\varphi_{i}\right\|^{2}-4 \sum_{j=1}^{k} a_{i j} b_{i j}+\int_{D} 4 g u_{i}\left\langle\nabla g, \nabla\left(\Delta u_{i}\right)\right\rangle,
\end{aligned}
$$

where

$$
b_{i j}=\int_{D}\left\langle\nabla g, \nabla u_{i}\right\rangle\left(\Delta u_{j}\right)=-b_{j i}
$$

and

$$
\left\|\varphi_{i}\right\|^{2}=\int_{D} \varphi_{i}^{2}
$$

By a simple calculation, we have, from (2.5),

$$
\begin{aligned}
& 4 \int_{D} g u_{i}\left\langle\nabla g, \nabla\left(\Delta u_{i}\right)\right\rangle \\
= & -2 \int_{D} \Delta u_{i}\left\langle\nabla u_{i}, \nabla g^{2}\right\rangle-2 \int_{D} \Delta u_{i}\left(u_{i} \Delta g^{2}\right) \\
= & \int_{D}\left(4\left|\nabla_{p} u_{i}\right|^{2}+2\left|\nabla u_{i}\right|^{2}\right)=4\left\|\nabla_{p} u_{i}\right\|^{2}+2\left\|\nabla u_{i}\right\|^{2} .
\end{aligned}
$$

Then, according to $(2.4),(2.6)$ and (2.8), we obtain

$$
\left(\lambda_{k+1}-\lambda_{i}\right)\left\|\varphi_{i}\right\|^{2} \leq\left(2\left\|\nabla u_{i}\right\|^{2}+4\left\|\nabla_{p} u_{i}\right\|^{2}\right)-4 \sum_{j=1}^{k} a_{i j} b_{i j} .
$$

On the other hand, since

$$
\int_{D} u_{i}\left\langle\nabla\left(g u_{i}\right), \nabla g\right\rangle=\frac{1}{2}
$$


holds, we have

$$
\begin{aligned}
& \int_{D} \varphi_{i}\left(-2\left\langle\nabla g, \nabla u_{i}\right\rangle\right) \\
= & -2 \int_{D}\left(g u_{i}-\sum_{j=1}^{k} a_{i j} u_{j}\right)\left\langle\nabla g, \nabla u_{i}\right\rangle \\
= & 2 \int_{D} u_{i}\left\langle\nabla\left(g u_{i}\right), \nabla g\right\rangle+2 \int_{D} \sum_{j=1}^{k} a_{i j} u_{j}\left\langle\nabla g, \nabla u_{i}\right\rangle \\
= & 1+2 \sum_{j=1}^{k} a_{i j} c_{i j},
\end{aligned}
$$

where

$$
c_{i j}=\int_{D} u_{j}\left\langle\nabla g, \nabla u_{i}\right\rangle=-c_{j i}
$$

Because of

$$
\lambda_{i} a_{i j}=\int_{D}\left(\Delta^{2} u_{i}\right) g u_{j}=\lambda_{j} a_{i j}+4 \int_{D}\left\langle\nabla g, \nabla u_{j}\right\rangle\left(\Delta u_{i}\right)=\lambda_{j} a_{i j}-4 b_{i j},
$$

we have

$$
-\left(\lambda_{i}-\lambda_{j}\right) a_{i j}=4 b_{i j}=-4 b_{j i}
$$

For any constant $\alpha>0$, we have, from (2.3), (2.5) and (2.10),

$$
\begin{aligned}
& 1+2 \sum_{j=1}^{k} a_{i j} c_{i j} \\
= & \int_{D} \varphi_{i}\left(-2\left\langle\nabla g, \nabla u_{i}\right\rangle+2 \sum_{j=1}^{k} c_{i j} u_{j}\right) \\
\leq & \int_{D}\left\{\alpha \varphi_{i}^{2}+\frac{1}{\alpha}\left(-\left\langle\nabla g, \nabla u_{i}\right\rangle+\sum_{j=1}^{k} c_{i j} u_{j}\right)^{2}\right\} \\
= & \alpha\left\|\varphi_{i}\right\|^{2}+\frac{1}{\alpha}\left(\left\|\nabla_{p} u_{i}\right\|^{2}-\sum_{j=1}^{k} c_{i j}^{2}\right) .
\end{aligned}
$$


Multiplying (2.13) by $\left(\lambda_{k+1}-\lambda_{i}\right)$, we infer, from (2.9),

$$
\begin{aligned}
& \left(1+2 \sum_{j=1}^{k} a_{i j} c_{i j}\right)\left(\lambda_{k+1}-\lambda_{i}\right) \\
\leq & \left(\lambda_{k+1}-\lambda_{i}\right)\left\{\alpha\left\|\varphi_{i}\right\|^{2}+\frac{1}{\alpha}\left(\left\|\nabla_{p} u_{i}\right\|^{2}-\sum_{j=1}^{k} c_{i j}^{2}\right)\right\} \\
\leq & \alpha\left(2\left\|\nabla u_{i}\right\|^{2}+4\left\|\nabla_{p} u_{i}\right\|^{2}-4 \sum_{j=1}^{k} a_{i j} b_{i j}\right) \\
& +\frac{\lambda_{k+1}-\lambda_{i}}{\alpha}\left(\left\|\nabla_{p} u_{i}\right\|^{2}-\sum_{j=1}^{k} c_{i j}^{2}\right) .
\end{aligned}
$$

Putting $\alpha=\left(\lambda_{k+1}-\lambda_{i}\right)^{1 / 2} \alpha_{1}, \alpha_{1}=(2 n+4)^{-1 / 2}$, we have

$$
\begin{aligned}
& \lambda_{k+1}-\lambda_{i}+2 \sum_{j=1}^{k}\left(\lambda_{k+1}-\lambda_{i}\right) a_{i j} c_{i j} \\
& \leq \alpha_{1}\left(\lambda_{k+1}-\lambda_{i}\right)^{1 / 2}\left(2\left\|\nabla u_{i}\right\|^{2}+4\left\|\nabla_{p} u_{i}\right\|^{2}-4 \sum_{j=1}^{k} a_{i j} b_{i j}\right) \\
& +\frac{1}{\alpha_{1}}\left(\lambda_{k+1}-\lambda_{i}\right)^{1 / 2}\left(\left\|\nabla_{p} u_{i}\right\|^{2}-\sum_{j=1}^{k} c_{i j}^{2}\right) .
\end{aligned}
$$

Taking sum on $i$ from 1 to $k$ for (2.15), we have

$$
\begin{aligned}
& \sum_{i=1}^{k}\left(\lambda_{k+1}-\lambda_{i}\right)+2 \sum_{i=1}^{k} \sum_{j=1}^{k}\left(\lambda_{k+1}-\lambda_{i}\right) a_{i j} c_{i j} \\
& \leq \alpha_{1} \sum_{i=1}^{k}\left(\lambda_{k+1}-\lambda_{i}\right)^{1 / 2}\left(2\left\|\nabla u_{i}\right\|^{2}+4\left\|\nabla_{p} u_{i}\right\|^{2}-4 \sum_{j=1}^{k} a_{i j} b_{i j}\right) \\
& +\frac{1}{\alpha_{1}} \sum_{i=1}^{k}\left(\lambda_{k+1}-\lambda_{i}\right)^{1 / 2}\left(\left\|\nabla_{p} u_{i}\right\|^{2}-\sum_{j=1}^{k} c_{i j}^{2}\right)
\end{aligned}
$$

Defining

$$
A=\sum_{i=1}^{k}\left(\lambda_{k+1}-\lambda_{i}\right)^{1 / 2}\left[\alpha_{1}\left(4\left\|\nabla_{p} u_{i}\right\|^{2}+2\left\|\nabla u_{i}\right\|^{2}\right)+\frac{1}{\alpha_{1}}\left\|\nabla_{p} u_{i}\right\|^{2}\right],
$$

we have

$$
\begin{aligned}
& \sum_{i=1}^{k}\left(\lambda_{k+1}-\lambda_{i}\right)+2 \sum_{i=1}^{k} \sum_{j=1}^{k}\left(\lambda_{k+1}-\lambda_{i}\right) a_{i j} c_{i j} \\
\leq & A-4 \alpha_{1} \sum_{i=1}^{k} \sum_{j=1}^{k}\left(\lambda_{k+1}-\lambda_{i}\right)^{1 / 2} a_{i j} b_{i j}-\frac{1}{\alpha_{1}} \sum_{i=1}^{k} \sum_{j=1}^{k}\left(\lambda_{k+1}-\lambda_{i}\right)^{1 / 2} c_{i j}^{2} .
\end{aligned}
$$


Since $a_{i j}=a_{j i}, c_{i j}=-c_{j i}$, we have

$$
2 \sum_{i=1}^{k} \sum_{j=1}^{k}\left(\lambda_{k+1}-\lambda_{i}\right) a_{i j} c_{i j}=-\sum_{i=1}^{k} \sum_{j=1}^{k}\left(\lambda_{i}-\lambda_{j}\right) a_{i j} c_{i j} .
$$

From (2.12), we have

$$
4 b_{i j}=-\left(\lambda_{i}-\lambda_{j}\right) a_{i j} .
$$

Thus, we can obtain

$$
\begin{aligned}
& -4 \alpha_{1} \sum_{i=1}^{k} \sum_{j=1}^{k}\left(\lambda_{k+1}-\lambda_{i}\right)^{1 / 2} a_{i j} b_{i j} \\
= & \alpha_{1} \sum_{i=1}^{k} \sum_{j=1}^{k}\left(\lambda_{k+1}-\lambda_{i}\right)^{1 / 2}\left(\lambda_{i}-\lambda_{j}\right) a_{i j}^{2} \\
= & \frac{\alpha_{1}}{2} \sum_{i=1}^{k} \sum_{j=1}^{k}\left\{\left(\lambda_{k+1}-\lambda_{i}\right)^{1 / 2}-\left(\lambda_{k+1}-\lambda_{j}\right)^{1 / 2}\right\}\left(\lambda_{i}-\lambda_{j}\right) a_{i j}^{2} \\
= & -\frac{\alpha_{1}}{2} \sum_{i=1}^{k} \sum_{j=1}^{k} \frac{1}{\left(\lambda_{k+1}-\lambda_{i}\right)^{1 / 2}+\left(\lambda_{k+1}-\lambda_{j}\right)^{1 / 2}}\left(\lambda_{i}-\lambda_{j}\right)^{2} a_{i j}^{2}
\end{aligned}
$$

and

$$
\begin{aligned}
& -\frac{1}{\alpha_{1}} \sum_{i=1}^{k} \sum_{j=1}^{k}\left(\lambda_{k+1}-\lambda_{i}\right)^{1 / 2} c_{i j}^{2} \\
= & -\frac{1}{2 \alpha_{1}} \sum_{i=1}^{k} \sum_{j=1}^{k}\left\{\left(\lambda_{k+1}-\lambda_{i}\right)^{1 / 2}+\left(\lambda_{k+1}-\lambda_{j}\right)^{1 / 2}\right\} c_{i j}^{2} .
\end{aligned}
$$

Since

$$
\begin{aligned}
& \sum_{i=1}^{k} \sum_{j=1}^{k}\left(\lambda_{i}-\lambda_{j}\right) a_{i j} c_{i j} \\
\leq & \frac{\alpha_{1}}{2} \sum_{i=1}^{k} \sum_{j=1}^{k} \frac{1}{\left(\lambda_{k+1}-\lambda_{i}\right)^{1 / 2}+\left(\lambda_{k+1}-\lambda_{j}\right)^{1 / 2}}\left(\lambda_{i}-\lambda_{j}\right)^{2} a_{i j}^{2} \\
+ & \frac{1}{2 \alpha_{1}} \sum_{i=1}^{k} \sum_{j=1}^{k}\left\{\left(\lambda_{k+1}-\lambda_{i}\right)^{1 / 2}+\left(\lambda_{k+1}-\lambda_{j}\right)^{1 / 2}\right\} c_{i j}^{2}
\end{aligned}
$$

holds, we infer, from (2.18), (2.19), (2.20) and (2.21),

$$
\sum_{i=1}^{k}\left(\lambda_{k+1}-\lambda_{i}\right) \leq A \text {. }
$$

That is,

$$
k \lambda_{k+1}-\sum_{i=1}^{k} \lambda_{i} \leq A .
$$


From the definition of $A$ and $\alpha_{1}=(2 n+4)^{-\frac{1}{2}}$, we obtain, by taking the sum on $p$ from 1 to $n$ for (2.22),

$$
\begin{aligned}
n\left(k \lambda_{k+1}-\sum_{i=1}^{k} \lambda_{i}\right) & \leq \sum_{i=1}^{k}\left(\lambda_{k+1}-\lambda_{i}\right)^{1 / 2}\left[\alpha_{1}(2 n+4)+\frac{1}{\alpha_{1}}\right]\left\|\nabla u_{i}\right\|^{2} \\
& =[8(n+2)]^{1 / 2} \sum_{i=1}^{k}\left(\lambda_{k+1}-\lambda_{i}\right)^{1 / 2}\left\|\nabla u_{i}\right\|^{2} .
\end{aligned}
$$

Since

$$
\left\|\nabla u_{i}\right\|^{2}=\int_{D}\left|\nabla u_{i}\right|^{2}=\int_{D} u_{i}\left(-\Delta u_{i}\right) \leq\left(\left\|u_{i}\right\|^{2}\left\|\Delta u_{i}\right\|^{2}\right)^{1 / 2}=\left(\lambda_{i}\right)^{1 / 2},
$$

we have

$$
k \lambda_{k+1}-\sum_{i=1}^{k} \lambda_{i} \leq \frac{[8(n+2)]^{1 / 2}}{n} \sum_{i=1}^{k}\left[\left(\lambda_{k+1}-\lambda_{i}\right) \lambda_{i}\right]^{1 / 2} .
$$

This completes the proof of Theorem 1 .

We now prove Corollary 1.

Proof of Corollary 1. Let

$$
\Lambda_{k}=\frac{1}{k} \sum_{i=1}^{k} \lambda_{i}, \quad T_{k}=\frac{1}{k} \sum_{i=1}^{k} \lambda_{i}^{2} .
$$

It follows from (2.25) that

$$
\begin{aligned}
& \left(\lambda_{k+1}-\Lambda_{k}\right)^{2} \\
\leq & \frac{8(n+2)}{n^{2}}\left[\frac{1}{k} \sum_{i=1}^{k}\left\{\left(\lambda_{k+1}-\lambda_{i}\right) \lambda_{i}\right\}^{1 / 2}\right]^{2} \\
\leq & \frac{8(n+2)}{n^{2}} \frac{1}{k} \sum_{i=1}^{k}\left(\lambda_{k+1}-\lambda_{i}\right) \lambda_{i} \\
= & \frac{8(n+2)}{n^{2}}\left(\lambda_{k+1} \Lambda_{k}-T_{k}\right) .
\end{aligned}
$$

Therefore,

$$
\begin{aligned}
& \left\{\lambda_{k+1}-\left[1+\frac{4(n+2)}{n^{2}}\right] \Lambda_{k}\right\}^{2} \\
\leq & {\left[\frac{8(n+2)}{n^{2}}+\frac{16(n+2)^{2}}{n^{4}}\right] \Lambda_{k}^{2}-\frac{8(n+2)}{n^{2}} T_{k} . }
\end{aligned}
$$

Namely,

$$
\begin{aligned}
& \lambda_{k+1}-\left[1+\frac{4(n+2)}{n^{2}}\right] \Lambda_{k} \\
& \leq\left\{\left[\frac{4(n+2)}{n^{2}} \Lambda_{k}\right]^{2}-\frac{8(n+2)}{n^{2}} \frac{1}{k} \sum_{j=1}^{k}\left(\lambda_{j}-\Lambda_{k}\right)^{2}\right\}^{1 / 2} .
\end{aligned}
$$

This finishes the proof of Corollary 1. 
Remark 2. In order to prove our Theorem 1, we introduced a factor $\left(\lambda_{k+1}-\lambda_{i}\right)$ in the formula (2.14). From the assertions from formulas (2.14) to (2.22), we know that unwanted terms between both sides of the inequalities are eliminated perfectly. If we do not introduced the factor $\left(\lambda_{k+1}-\lambda_{i}\right)$ in the formula (2.14), we shall obtain the inequality (2.10) of Hook, and Chen and Qian. In fact, putting $\alpha=\frac{\left(\lambda_{k+1}-\lambda_{i}\right) \alpha_{2}}{\sum_{l=1}^{k} \lambda_{l}^{\frac{1}{2}}}$, $\alpha_{2}=\frac{n k}{4(n+2)}$ and taking the sum on $i$ from 1 to $k$ for (2.13), we have, from (2.9),

$$
\begin{aligned}
& k+2 \sum_{i, j=1}^{k} a_{i j} c_{i j} \\
\leq & \frac{\alpha_{2}}{\sum_{l=1}^{k} \lambda_{l}^{\frac{1}{2}}}\left\{2 \sum_{i=1}^{k}\left(\left\|\nabla u_{i}\right\|^{2}+4\left\|\nabla_{p} u_{i}\right\|^{2}\right)+\sum_{i, j=1}^{k}\left(\lambda_{i}-\lambda_{j}\right) a_{i j}^{2}\right\} \\
+ & \frac{\sum_{l=1}^{k} \lambda_{l}^{\frac{1}{2}}}{\alpha_{2}} \sum_{i=1}^{k} \frac{1}{\lambda_{k+1}-\lambda_{i}}\left(\left\|\nabla_{p} u_{i}\right\|^{2}-\sum_{j=1}^{k} c_{i j}^{2}\right)
\end{aligned}
$$

where we used the formula (2.12). From the antisymmetry of $c_{i j}$ and $\left(\lambda_{i}-\lambda_{j}\right) a_{i j}^{2}$, we have

$$
2 \sum_{i, j=1}^{k} a_{i j} c_{i j}=0 \text { and } \sum_{i, j=1}^{k}\left(\lambda_{i}-\lambda_{j}\right) a_{i j}^{2}=0 .
$$

Making use of the similar assertion in the proof of Theorem 1, we have

$$
n k \leq 2(n+2) \alpha_{2}+\frac{1}{\alpha_{2}}\left[\sum_{i=1} \frac{\lambda_{i}^{1 / 2}}{\lambda_{k+1}-\lambda_{i}}\right] \sum_{i=1}^{k} \lambda_{i}^{1 / 2}-\frac{\sum_{l=1}^{k} \lambda_{l}^{\frac{1}{2}}}{\alpha_{2}} \sum_{i, j=1}^{k} \frac{c_{i j}^{2}}{\lambda_{k+1}-\lambda_{i}}
$$

Hence, we have

$$
\frac{n^{2} k^{2}}{8(n+2)} \leq\left[\sum_{i=1} \frac{\lambda_{i}^{1 / 2}}{\lambda_{k+1}-\lambda_{i}}\right] \sum_{i=1}^{k} \lambda_{i}^{1 / 2}-\sum_{l=1}^{k} \lambda_{l}^{\frac{1}{2}} \sum_{i, j=1}^{k} \frac{c_{i j}^{2}}{\lambda_{k+1}-\lambda_{i}}
$$

Therefore, in order to infer the inequality (1.10), we must throw away the unwanted term in (2.31). Thus, we know that the inequality in Theorem 1 should be sharper than (1.10). If we multiply $(2.13)$ by a factor $\left(\lambda_{k+1}-\lambda_{i}\right)^{\frac{1}{2}}$ and use a similar argument, we shall obtain the inequality (1.14).

\section{ACKNOWLEDGEMENTS}

This work was mainly done while the second author was visiting Saga University. He was supported in part by a Grant-in-Aid for Scientific Research from the Japan Society for the Promotion of Science. He would like to express his gratitude to Professor Qing-Ming Cheng for his kind hospitality.

\section{REFERENCES}

[1] M. S. Ashbaugh, Isoperimetric and universal inequalities for eigenvalues, in Spectral theory and geometry (Edinburgh, 1998), E. B. Davies and Yu Safalov eds., London Math. Soc. Lecture Notes, vol. 273, Cambridge Univ. Press, Cambridge, 1999, pp. 95-139. MR1736867 (2001a:35131)

[2] M. S. Ashbaugh, Universal eigenvalue bounds of Payne-Pólya-Weinberger, Hile-Protter and H.C. Yang, Proc. Indian Acad. Sci. Math. Sci. 112 (2002), 3-30. MR1894540 (2004c:35302) 
[3] M. S. Ashbaugh and R. D. Benguria, Proof of the Payne-Pólya-Weinberger conjecture, Bull. Amer. Math. Soc. 25 (1991), 19-29. MR1085824 (91m:35173)

[4] M. S. Ashbaugh and R. D. Benguria, A sharp bound for the ratio of the first two eigenvalues of Dirichlet Laplacians and extensions, Ann. of Math. 135 (1992), 601-628. MR:1166646 (93d:35105)

[5] M. S. Ashbaugh and R. D. Benguria, A second proof of the Payne-Pólya-Weinberger conjecture, Commun. Math. Phys. 147 (1992), 181-190. MR.1171765 (93k:33002)

[6] Z.-C. Chen and C.-L. Qian, Estimates for discrete spectrum of Laplacian operator with any order, J. China Univ. Sci. Tech. 20 (1990), 259-266. MR1077287 (92c:35087)

[7] G. N. Hile and M. H. Protter, Inequalities for eigenvalues of the Laplacian, Indiana Univ. Math. J. 29 (1980), 523-538. MR0578204 (82c:35052)

[8] G. N. Hile and R. Z. Yeh, Inequalities for eigenvalues of the biharmonic operator, Pacific J. Math. 112 (1984), 115-133. MR0739143 (85k:35170)

[9] S. M. Hook, Domain independent upper bounds for eigenvalues of elliptic operator, Trans. Amer. Math. Soc. 318 (1990), 615-642. MR0994167 (90h:35075)

[10] L. E. Payne, G. Polya, and H. F. Weinberger, Sur le quotient de deux fréquences propres consécutives, Comptes Rendus Acad. Sci. Paris 241 (1955), 917-919. MR0073046 (17:372d)

[11] L. E. Payne, G. Polya, and H. F. Weinberger, On the ratio of consecutive eigenvalues, J. Math. and Phys. 35 (1956), 289-298. MR0084696 (18:905c)

[12] H. C. Yang, An estimate of the differance between consecutive eigenvalues, preprint IC/91/60 of ICTP, Trieste, 1991.

Department of Mathematics, Faculty of Science and Engineering, Saga University, SAGA $840-8502$, JAPAN

E-mail address: cheng@ms.saga-u.ac.jp

Academy of Mathematics and Systematical Sciences, CAS, Beijing 100080, People's Republic of China

E-mail address: yanghc@math03.math.ac.cn 